\title{
Inferior STEMI complicated by retroperitoneal hemorrhage due to ruptured AAA
}

Farley B. Neasman III, MD ${ }^{1}$, Melissa D. Lester, DO ${ }^{1}$, Nepal C. Chowdhury MD²

Author Affiliations:

1. Marshall University, Huntington, West Virginia

2. St. Mary's Medical Center Cardiothoracic Surgery, Huntington, West Virginia

The authors have no financial disclosures to declare and no conflicts of interest to report.

\section{Corresponding Author:}

Farley B. Neasman III, MD Marshall University

Huntington, West Virginia

Email: farley3@gmail.com 


\section{Abstract}

When patients present with life threatening conditions, a rapid cost-benefit analysis prioritizes care and commits treatment to a certain course that, in the case of ST Elevation Myocardial Infarction (STEMI) treated with drug-eluting stents (DES), could be fatal if there is any deviation. Antiplatelet therapy is vital and secondary concerns (i.e. bleeding diatheses) may accept suboptimal outcomes - in rare cases, another life-threatening condition may be unmasked, the treatment for which runs directly counter to the first. We present a case of STEMI with high clot burden treated with multiple DES, complicated by retroperitoneal hemorrhage due to a ruptured abdominal aortic aneurysm.

\section{Keywords}

STEMI, retroperitoneal hemorrrhage, abdominal aortic aneurysm, anticoagulation

\section{Introduction}

A 68 year old Caucasian male with a past medical history of coronary artery disease (CAD) and prior myocardial infarction treated with percutaneous coronary intervention (PCI), Vfib arrest, hypertension, hyperlipidemia, smoking and COPD presented to the emergency department following two hours of chest pain - EKG on admission revealed inferior Q waves and ST elevation. After initiation of heparin bolus and drip, he was taken for emergent left heat catheterization via right femoral access which revealed the culprit lesions to be a $90 \%$ proximal right coronary artery (RCA) stenosis with thrombus and 60-70\% in-stent restenosis (ISR). He received Xience Alpine 3.5x9mm and 3.0x12mm drug-eluting stents (DES) to his proximal RCA, and Xience alpine 3.0x12mm and 3.0x38mm DES to his mid-RCA.

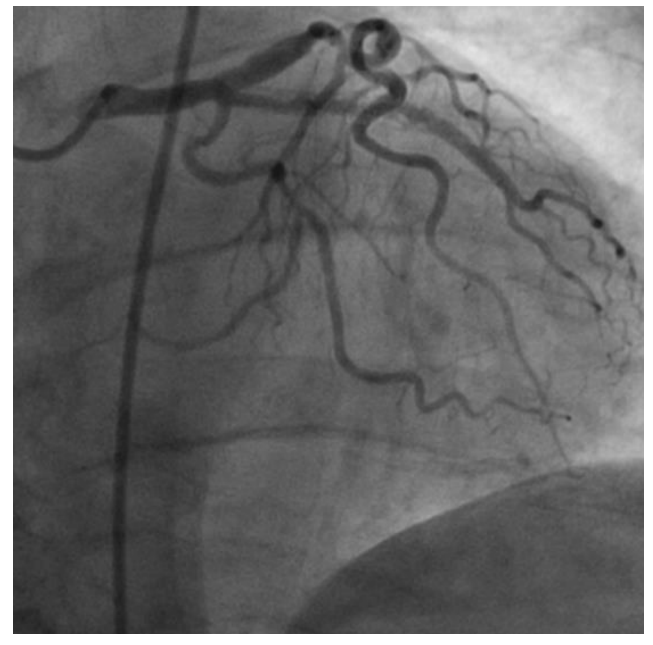

Figure A: left coronary system, AP projection

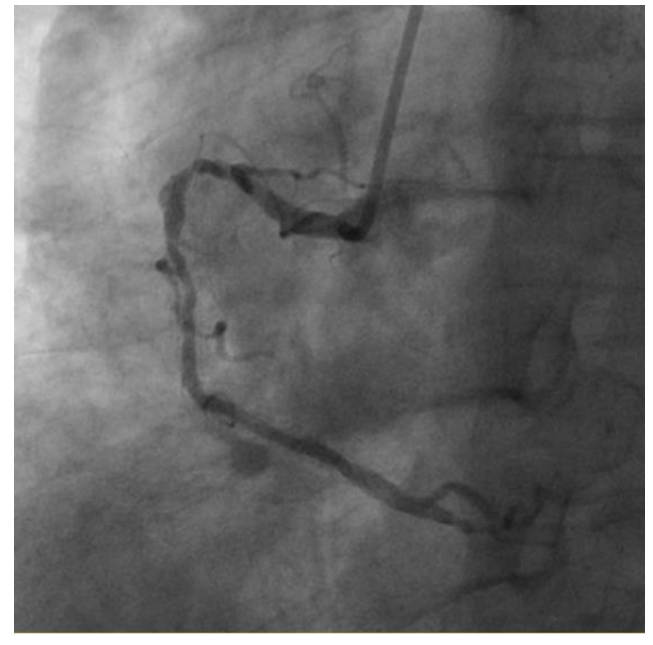

Figure B: Right coronary system, LAO projection 
He tolerated the procedure well and was transferred to the CCU on aspirin, Plavix and Integrilin, due to the thrombus burden. Six hours later, he began complaining of abdominal discomfort and received IV morphine and IV Dilaudid. He was then noted to have downward trending blood pressures - fluids were initiated, and due to concern for retroperitoneal hemorrhage, CT abdomen was ordered. In addition to a large retroperitoneal hematoma, CT revealed a ruptured infra-renal abdominal aortic aneurysm (AAA) $9.1 \mathrm{~cm}$ antero-posterior by $8.7 \mathrm{~cm}$ transverse by $12.1 \mathrm{~cm}$ in length, with interior features concerning for intramural hematoma vs. underlying dissection.
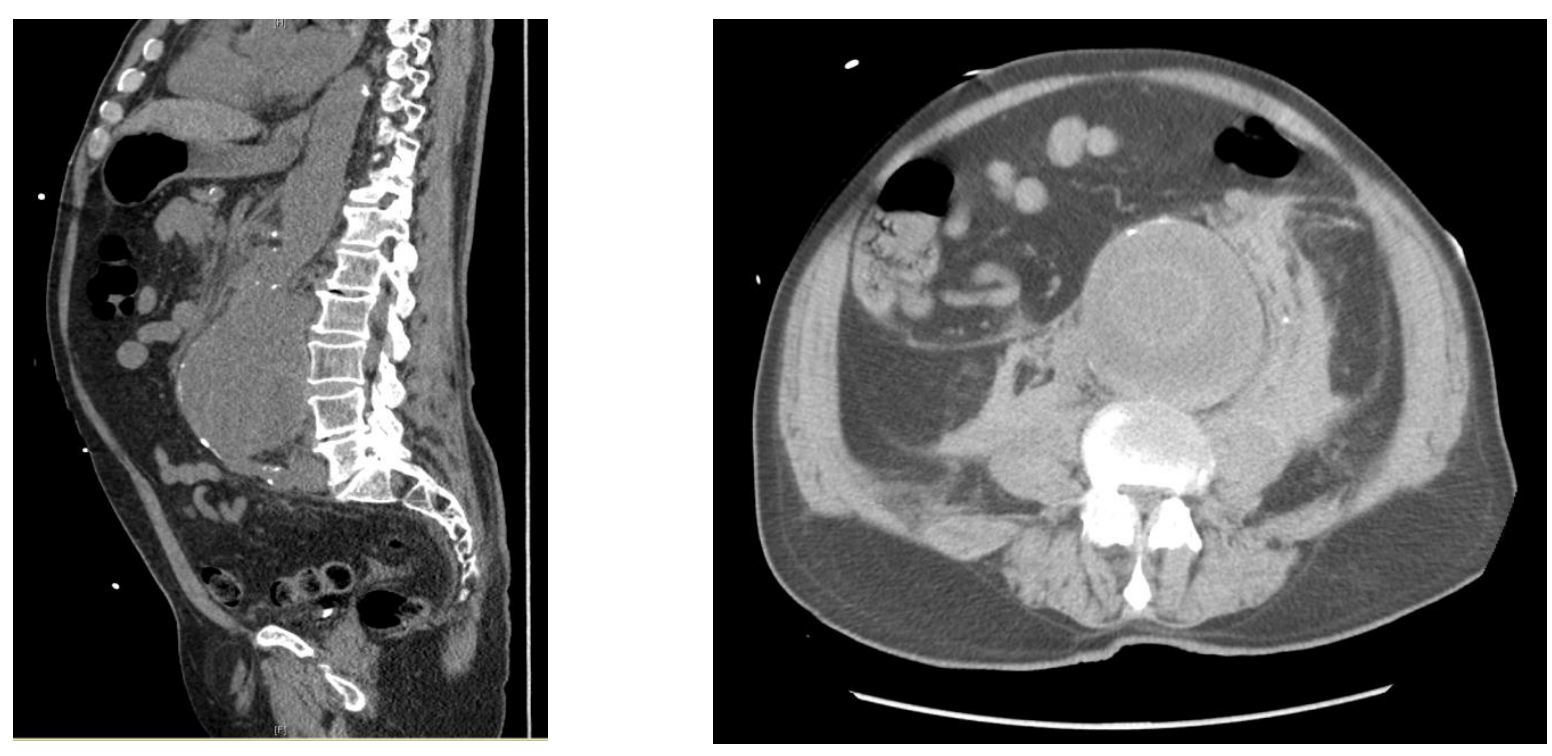

Figures C and D: Abdominal Aortic Aneurysm

An emergent call was placed to cardiothoracic / vascular surgery, and the patient was immediately evaluated. A brief discussion revealed that there was no graft available large enough for an endovascular repair (EVAR), and he was taken to the OR for emergent open AAA repair. During the procedure he was found to have thrombus and plaque within the aneurysm cavity with complete posterior and left lateral aneurysmal tear, and a $16 \mathrm{~mm}$ Hemashield graft was used for the repair. Hemoglobin $(\mathrm{Hb})$ on admission trended down from 11.5 to 9.7 immediately prior to the OR, he was transfused to a hemoglobin of 11.2, and this trended down to a nadir of 7.3 four days post-surgery. Dual antiplatelet therapy was by necessity continued, and he was again transfused, for a total of 8 units. Repeat CT abdomen revealed significant improvement in retroperitoneal hemorrhage, though he was found to be positive for fecal occult blood. He was evaluated by gastroenterology, upper endoscopy the following day revealed gastritis and a nonbleeding gastric ulcer, and he was started on a proton pump inhibitor. The rest of the patient's hospital course was complicated by atrial tachycardia, acute kidney injury, and confusion, all of which resolved with appropriate treatment. He was evaluated by physical therapy and inpatient rehab was recommended, though the patient declined and was ultimately discharged in good health, with $\mathrm{Hb}$ over 8 . He did not keep his follow-up visit with cardiology. 


\section{Discussion}

"There is no disease more conducive to clinical humility than aneurysm of the aorta."

- Sir William Osler

More remarkable than this patient's clinical course is the fact that he survived to discharge. Heart disease is responsible for 1 in 4 deaths in the US, claiming 670,000 lives - 61\% of which are attributable to coronary heart disease. ${ }^{1}$ Although this patient presented as a STEMI, it was in his favor that he was not in cardiogenic shock and didn't require mechanical support. However, his stent placement was not without subsequent risk; one review found that with ideal compliance with dual antiplatelet therapy (DAPT), the incidence of definite stent thrombosis at 3 years was $1 \%$ with $2^{\text {nd }}$ generation DES, $1.5 \%$ with bare metal stents (BMS) and $2.2 \%$ with first generation DES. ${ }^{2}$ Though the updated recommendations on the duration of antiplatelet therapy following stent placement were relaxed to a minimum of 6 months in stable ischemic heart disease, it remains a class I recommendation to continue DAPT for a minimum of 12 months in the setting of acute coronary syndromes. ${ }^{3}$ A meta-analysis found that the risk of stent thrombosis was highest in the setting of extensive CAD, early discontinuation of DAPT, and number of stents ${ }^{4}$ with these risk factors alone, the patient was already at higher risk for a negative outcome.

Patients frequently experience post-catheterization groin or back discomfort, though it was this patient's downward trending blood pressure that ultimately revealed the cause of his discomfort. Retroperitoneal hemorrhage (RPH) is a feared complication of cardiac catheterization via femoral access, with prevalence ranging from $0.4 \%$ to $0.74 \% .^{5-7}$ Features include groin pain in $42-100 \%$, back pain in 23-64\%, and hypotension in 92\%. Female gender, low body surface area, and high arterial puncture are recognized risk factors; one review found no association between RPH and sheath size, GpIIb/IIIa inhibition, and use of a closure device ${ }^{5}$, though others have shown a link between sheath size and use of closure device ${ }^{7}$. RPH is associated with high inhospital mortality $(6.64 \%$ vs. $1.07 \%)$ in those without RPH, due in part to the often insidious presentation. ${ }^{7}$ The majority of patients (84-92.3\%) with RPH are treat medically, with few requiring surgery ${ }^{5,7}$, though placement of a covered stent is an acceptable treatment with favorable anatomy. In one review the average drop in hemoglobin among patients with RPH was $11.5 \pm 5$ s compared with $2.3 \pm 3.3$ in controls. ${ }^{6}$ The previous statistics cannot apply to this patient, however; his RPH was determined to be due to his ruptured AAA, which carried a far more dismal prognosis.

Patients with CAD are often at risk for AAA; one review found the incidence of AAA in patients over 60 with CAD to be $14 \%$, as compared to $3 \%$ in patients without CAD. ${ }^{8}$ Smoking was found to be a strong risk factor for not only AAA development but also rupture ${ }^{9}$, though diabetes mellitus was found to be negatively associated with AAA. The USPSTF recommends a one-time screening abdominal ultrasound in men between the ages of 65 and 75 who have ever smoked, with a class $B$ recommendation (strong evidence that the effect is moderate, or moderate effect that the evidence is strong).$^{10}$ The prevalence of AAA is estimated to be $1.3 \%$ in women and 5- 
$9 \%$ in men over $65^{11,12}$, with mortality due to ruptured AAA as high as $90 \%{ }^{13}$, making ruptured AAA the $13^{\text {th }}$ leading cause of death in the western world as of $2005^{14}$. Further complicating matters, open AAA repair is associated with a $53 \%$ mortality, compared to $24 \%$ with endovascular aneurysm repair (EVAR). ${ }^{15}$

The conflict at the crux of this patient's care was the aggressive anticoagulation necessary to maintain patency of multiple drug-eluting stents, and the effect of that anticoagulation on his potentially catastrophic AAA. Did the LHC contribute to aneurysmal rupture? It is certainly a possibility; advancement of the catheters could have caused trauma to an already friable endothelium. Nevertheless, the patient was already at risk prior to his LHC. Application of Laplace's law to aneurysms (increasing wall tension with increasing diameter) has been found to be faulty due to aneurysm wall heterogeneity, with different segments of the aneurysmal wall experiencing different wall stress - thus, smaller aneurysms area also at risk of rupture. ${ }^{14}$ However, the recommendation for repair at $5 \mathrm{~cm}$ is due to the increased likelihood of rupture, with a $32.5 \% 1$ year incidence of rupture for AAA $>7 \mathrm{~cm} .{ }^{16}$ Thus, it is entirely possible that this patient's AAA had been slowly bleeding prior to his STEMI, and that the administration of aspirin, Plavix, and Integrilin hastened the process. Essentially, the only options were either to discontinue all antiplatelet / anticoagulant medications in favor of reducing bleeding from the AAA, or to continue DAPT and hope for the best, with the former option having been determined to have worse early and late outcomes than the latter. Ultimately, despite continued DAPT, the patient's bleeding abated and his surgical site remained stable - even his subsequent GI bleed required no intervention. The close communication between interventional cardiology and cardiothoracic / vascular surgery led to decisive, timely treatment, setting the stage for an optimal outcome against overwhelming odds. 


\section{References}

1. CDC, NCHS. Underlying Cause of Death 1999-2013 on CDC WONDER Online Database, released 2015. Data are from the Multiple Cause of Death Files, 1999-2013, as compiled from data provided by the 57 vital statistics jurisdictions through the Vital Statistics Cooperative Program. Accessed Feb. 3, 2015.

2. Tada T, Byrne RA, Simunovic I et al. Risk of stent thrombosis among bare-metal stents, first-generation drug-eluting stents, and second-generation drug-eluting stents: results from a registry of 18,334 Patients. $J$ Am Coll Cardiol Intv. 2013;6(12):1267-1274. doi:10.1016/j.jcin.2013.06.015

3. 2016 ACC/AHA guideline focused update on duration of dual antiplatelet therapy in patients with coronary artery disease: a report of the American College of Cardiology/American Heart Association task force on clinical practice guidelines. An update of the 2011 ACC/AHA/SCAI PCI Guideline, 2011 ACC/AHA CABG Guideline, 2012 ACCF/AHA/ACP/AATS/PCNA/SCAI/STS SIHD Guideline, 2013 ACC/AHA STEMI Guideline, 2014 ACC/AHA NSTE-ACS Guideline, and 2014 ACC/AHA Perioperative Guideline. J Am Coll Cardiol. 2016;Mar 29.

4. D'Ascenzo, Fabrizio et al. Incidence and predictors of coronary stent thrombosis: evidence from an international collaborative meta-analysis including 30 studies, 221,066 patients, and 4276 thromboses. International journal of cardiology. 167.2 (2013): 575-584.

5. Farouque H, Tremmel JA, Raissi Shabari F et al. Risk factors for the development of retroperitoneal hematoma after percutaneous coronary intervention in the era of glycoprotein IIb/IIIa inhibitors and vascular closure devices. J Am Coll Cardiol. 2005;45(3):363-368. doi:10.1016/j.jacc.2004.10.042.

6. Kent K. Craig et al. Retroperitoneal hematoma after cardiac catheterization: prevalence, risk factors, and optimal management. Journal of vascular surgery. 20.6 (1994): 905-913.

7. Trimarchi S, Smith DE, Share D et al. Retroperitoneal hematoma after percutaneous coronary intervention: prevalence, risk factors, management, outcomes, and predictors of mortality: a report from the BMC2 (Blue Cross Blue Shield of Michigan Cardiovascular Consortium) Registry. J Am Coll Cardiol Intv. 2010;3(8):845-850. doi:10.1016/j.jcin.2010.05.013.

8. Madaric Juraj et al. Frequency of abdominal aortic aneurysm in patients $>60$ years of age with coronary artery disease. The American journal of cardiology. 96.9 (2005): 1214-1216.

9. Strachan D. P. Predictors of death from aortic aneurysm among middle-aged men: the Whitehall study. British journal of surgery. 78.4 (1991): 401-404.

10. LeFevre Michael L. Screening for abdominal aortic aneurysm: US Preventive Services Task Force recommendation statement..Annals of internal medicine. 161.4 (2014): 281-290.

11. Scott RAP, Bridgewater S, Ashton HA. Randomised clinical trial of screening for abdominal aortic aneurysm in women. Br J Surg. 2002; 89: 283-285.

12. Thompson RW, Curci JA, Ennis TL et al. Pathophysiology of abdominal aortic aneurysms: insights from the elastase-induced model in mice with different genetic backgrounds. Ann N Y Acad Sci 2006;1085:59-7

13. Pearce William H, Christopher K. Zarins, J. Michael Bacharach. Atherosclerotic peripheral vascular disease symposium II controversies in abdominal aortic aneurysm repair. Circulation 118.25 (2008): 28602863.

14. Choke $\mathrm{E}$ et al. A review of biological factors implicated in abdominal aortic aneurysm rupture. European Journal of Vascular and Endovascular Surgery 30.3 (2005): 227-244.

15. Eefting D et al. Ruptured AAA: state of the art management. The Journal of cardiovascular surgery 54.1 Suppl 1 (2013): 47-53.

16. Lederle Frank A et al. Rupture rate of large abdominal aortic aneurysms in patients refusing or unfit for elective repair. Jama 287.22 (2002): 2968-2972. 